This document is the Accepted Manuscript version of a Published Work that appeared in final form in ACS Applied Materials and Interfaces, copyright (c) American Chemical Society after peer review and technical editing by the publisher. To access the final edited and published work see: https://dx.doi.org/10.1021/acsami.0c04331. 


\section{Tin Selenide Molecular Precursor for the Solution-Processing of Thermoelectric Materials and Devices}

Yu Zhang, ${ }^{\dagger, \#}$ Yu Liu, ${ }^{\not, \#}$ Congcong Xing, ${ }^{\dagger}, \S$ Ting Zhang, ${ }^{\perp}$ Mengyao Li, ${ }^{\dagger}$ Mercè Pacios, ${ }^{\dagger}$ Xiaoting

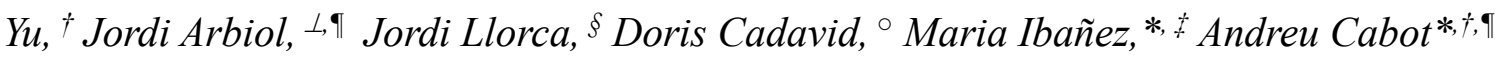

${ }^{\dagger}$ Catalonia Energy Research Institute - IREC, Sant Adrià de Besòs, 08930 Barcelona, Spain.

IST Austria, Am Campus 1, 3400 Klosterneuburg, Austria.

$\S$ Institute of Energy Technologies, Department of Chemical Engineering and Barcelona Research Center in Multiscale Science and Engineering, Universitat Politècnica de Catalunya, EEBE, 08019 Barcelona, Spain.

${ }^{\perp}$ Catalan Institute of Nanoscience and Nanotechnology (ICN2), CSIC and BIST, Campus UAB, Bellaterra, 08193 Barcelona, Catalonia, Spain.

${ }^{\circ}$ Departamento de Fisica, Universidad Nacional de Colombia 111321, Ciudad Universitaria, Bogota, Colombia.

๑ ICREA, Pg. Lluis Companys 23, 08010 Barcelona, Catalonia, Spain.

\# These authors contributed equally to this work.

*E-mails: A. Cabot:acabot@irec.cat; M. Ibañez: mibanez@ist.ac.at Keywords: molecular ink, SnSe, thermoelectricity, printing, nanomaterial. 


\begin{abstract}
In the present work, we report a solution-based strategy to produce crystallographically textured SnSe bulk nanomaterials and printed layers with optimized thermoelectric performance in the direction normal to the substrate. Our strategy is based on the formulation of a molecular precursor that can be continuously decomposed to produce a $\mathrm{SnSe}$ powder or printed into predefined patterns. The precursor formulation and decomposition conditions are optimized to produce pure phase 2D SnSe nanoplates. The printed layer and the bulk material obtained after hot press displays a clear preferential orientation of the crystallographic domains, resulting in ultralow thermal conductivity of $0.55 \mathrm{Wm}^{-1} \mathrm{~K}^{-1}$ in the direction normal to the substrate. Such textured nanomaterials present highly anisotropic properties, with best thermoelectric performance in plane, i.e. in the directions parallel to the substrate, which coincide with the crystallographic $b c$ plane of $\mathrm{SnSe}$. This is an unfortunate characteristic because thermoelectric devices are designed to create/harvest temperature gradients in the direction normal to the substrate. We further demonstrate that this limitation can be overcome with the introduction of small amounts of tellurium in the precursor. The presence of tellurium allows reducing the band gap, increase both charge carrier concentration and mobility, especially cross plane, with a minimal decrease of the Seebeck coefficient. These effects translate into record out of plane ZT values at $800 \mathrm{~K}$.
\end{abstract}




\section{INTRODUCTION}

Solid state thermoelectric (TE) devices offer a direct and solid state means of conversion between thermal and electrical energy, which makes them extremely appealing for a wide range of applications. ${ }^{1-8}$ However, the relatively low energy conversion efficiency and high manufacturing cost of current TE devices, which are in most cases designed for cooling applications, makes them cost-efficient only in few niche markets. ${ }^{1,9}$ Towards improving costefficiency of TE devices, it is imperative to: i) implement lower cost fabrication processes; ii) optimize device parameters for each particular application to maximize efficiency and minimize the amount of used material; iii) develop high performance and lower cost TE materials.

Thermoelectric performance is generally quantified using an adimensional figure of merit, $\mathrm{ZT}=\sigma \mathrm{S}^{2} \mathrm{~T} / \kappa$, where $\sigma, \mathrm{S}, \mathrm{T}$ and $\kappa$ are the electrical conductivity, Seebeck coefficient, absolute temperature and thermal conductivity, respectively. $3,7,{ }^{10}$ ENREF 7 Among the different families of TE materials proposed, ${ }^{2,4}$ some two dimensional chalcogenides exhibit the best TE performance. ${ }^{11-17} \mathrm{Bi}_{2} \mathrm{Te}_{3}$-based alloys provide the highest $\mathrm{ZT}$ values at temperatures around ambient, up to 1.96 at $420 \mathrm{~K},{ }^{16}$ and dominate the TE market. ${ }^{18-21}$ On the other hand, SnSe single crystals have recently achieved unprecedented record ZT values in the medium-high temperature range, 2.6 at $923 \mathrm{~K} .{ }^{13,}{ }^{22} \mathrm{SnSe}$ has a layered crystal structure with weak Van der Waals bonds along the $a$ axis and tight covalent bonds along the $b c$ plane, which results in highly anisotropic transport properties and an impressively low lattice thermal conductivity. Nonetheless, the high production cost and poor mechanical properties of SnSe single crystals limit their use in real applications. All of these have sparked much interest in producing polycrystalline SnSe with TE performance approaching that of SnSe single crystals. Owing to the anisotropic properties of the material, the optimization of polycrystalline SnSe generally requires producing crystallographic textured samples. ${ }^{23-35}$ In this direction, while severe plastic deformation techniques are conventionally used to produce textured polycrystalline materials, ${ }^{36,37}$ spark plasma sintering and hot press approaches, more commonly used to consolidate TE polycrystalline materials, are also able to produce textured materials when using an open dye to induce extrusion or when incorporating a liquid phase in the process. ${ }^{15,16}$, $38-42$

Beyond engineering TE materials with improved performance and lower cost, novel strategies to fabricate TE devices that are less labour-intensive and which allow a more rational use of the TE material need to be developed. One potential alternative strategy is ink-jet 
printing. The main advantages of printing techniques are low cost, potential to produce shape adaptable or flexible devices, control over the thickness of the TE material and high material use efficiency. While printing strategies are currently well developed and used in a large number of applications, their implementation in new technologies strongly relies on the formulation of proper functional inks. SnSe inks could be formulated from colloidal nanoparticles produced from the decomposition/reaction of a tin precursor with selenium oxide or trioctylphospine selenium at moderate temperatures as previously reported. ${ }^{43-47}$ But it is more convenient, in terms of cost and quality of the produced layers, to formulate them ionic or molecular precursors.

We report here a thiol-free SnSe molecular precursor, which can be used to produce bulk nanocrystalline SnSe or can be directly printed into SnSe layers. We study the decomposition conditions and demonstrate the possibility to produce pure phase $\mathrm{SnSe}$ at moderate temperatures. We further analyse here the processing conditions necessary to obtain crystallographically textured $\mathrm{SnSe}$ bulk nanomaterials and layers and characterize their anisotropic TE properties. Finally, the TE performance of the material in the direction normal to the layer substrate is optimized by including small amounts of tellurium within the precursor. 


\section{RESULT AND DISCUSSION}

To prepare a SnSe molecular ink, the tin and selenium precursors and the solvent were selected taking into account four main parameters: i) minimize cost; ii) direct the crystal morphology toward obtaining planar structures that facilitate posterior assembly into textured nanomaterials; iii) reach a compromise between solvent volatility, which has implications in toxicity and precursor stability, and decomposition temperature, which is a key parameters determining production costs; iv) reduce toxicity, avoiding the use of thiols and hydrazine. Taking into account these points, a $\mathrm{SnSe}$ precursor was prepared by dissolving $\mathrm{SnCl}_{2}$ and $\mathrm{SeO}_{2}$ in a combination of OAm and TOP. OAm played a double role, it reduced $\mathrm{Se}^{4+}$ to $\mathrm{Se}^{0}$ and it coordinated to $\mathrm{Sn}^{2+}$ to form a Sn-OAm complex, $\mathrm{Sn}(\mathrm{R}-\mathrm{NH})_{2} \cdot{ }^{48-50}$ TOP coordinated with $\mathrm{Se}^{0}$ to yield TOPSe ${ }^{51}$ (see Figure S1 in the supporting information, SI).

To analyse the product of its decomposition, the precursor was dropped onto a substrate heated at $420{ }^{\circ} \mathrm{C}$. Upon reaching the heated substrate, the precursor was rapidly decomposed and a black powder was produced. Large amounts of powder could be obtained by continuously injecting/spraying the molecular ink toward a heated receptacle/support (Figure S1). TEM and SEM characterization of the powder obtained from the decomposition of the precursor showed the presence of square-like plates with a lateral size of $4 \pm 1 \mu \mathrm{m}$ and a thickness of $90 \pm 20 \mathrm{~nm}$ (Figure 1a, b). HRTEM and XRD analyses demonstrated the powder to be highly crystalline and to display a unique crystallographic phase that matched that of orthorhombic SnSe (JCPDS NO. 00-048-1224, Pnma space group, Figure 1c, e). From the XRD pattern, the relatively strong intensity of the $2 \theta=31.0^{\circ}$ diffraction peak indexed as (400), indicated a preferential growth in the $b c$ plane, i.e. the plane perpendicular to the [100] crystallographic direction. UVVis spectrum showed the SnSe powder to have an indirect band gap at $0.96 \mathrm{eV}$ (Figure S2).

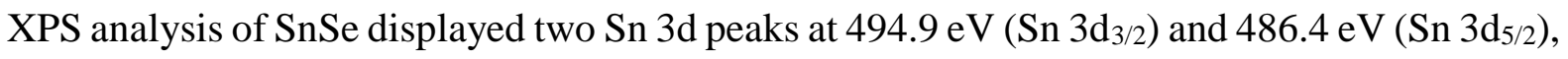
which matched well with a $\mathrm{Sn}^{2+}$ environment (Figure 1f). Besides, Se $3 \mathrm{~d}_{3 / 2}$ and $\mathrm{Se} 3 \mathrm{~d}_{5 / 2}$ peaks located at $54.4 \mathrm{eV}$ and $53.5 \mathrm{eV}$ evidenced the presence of a $\mathrm{Se}^{2-}$ oxidation state. EDX chemical analysis showed the SnSe powder to be slightly $\mathrm{Sn}$ rich, with a composition ratio $\mathrm{Se} / \mathrm{Sn}=0.95$ (Figure S3). EELS chemical composition maps displayed a homogeneously distribution of both Sn and Se within each particle and from particle to particle (Figure 1d).

To obtain single phase $\mathrm{SnSe}$, the proper adjustment of the nominal element ratio, $\mathrm{Sn} / \mathrm{Se}$, and decomposition temperature was fundamental. When increasing the nominal amount of Sn in the precursor formulation, a combination of $\mathrm{Sn}$ and $\mathrm{SnSe}$ phases was obtained upon decomposition at $420^{\circ} \mathrm{C}$ (Figures 2a). On the other hand, when using a nominal ratio $\mathrm{Sn} / \mathrm{Se}<1$, 
a combination of $\mathrm{SnSe}$ and $\mathrm{SnSe} \mathrm{S}_{2}$ was produced. Using a nominal $\mathrm{Sn} / \mathrm{Se}=0.5$ and decreasing the decomposition temperature to $360^{\circ} \mathrm{C}$, pure-phase $\mathrm{SnSe}_{2}$ could be produced (Figure 2a). Figure S4 shows XRD patterns of the materials obtained at different temperatures using a nominal element ratio $\mathrm{Sn} / \mathrm{Se}=1$.
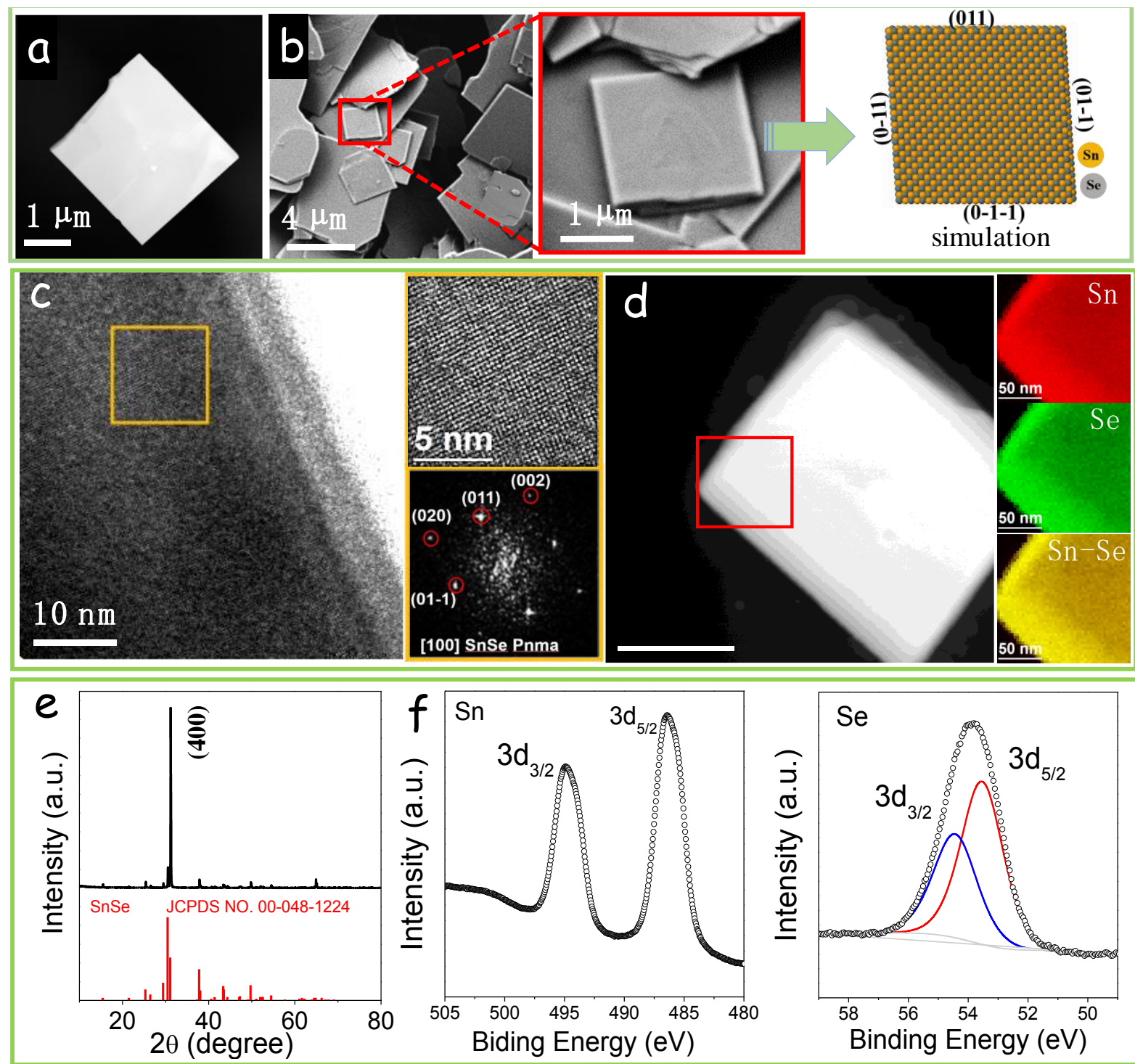

Figure 1. (a) Representative TEM and (b) SEM micrographs of square-like SnSe nanostructures, and a structure model of an individual nanoplate. (c) HRTEM micrograph of a SnSe nanoplate, magnified detail of the orange squared region and its corresponding power spectrum. The SnSe lattice fringe distances were measured to be $0.304,0.207 \mathrm{~nm}$ and $0.302 \mathrm{~nm}$, at 43.53 , and $87.51^{\circ}$, which was interpreted as the orthorhombic SnSe phase, visualized along its [100] zone axis. (d) EELS chemical composition maps obtained from the red squared area of the STEM micrograph. Individual Sn $\mathrm{M}_{4,5-}$ edges at $485 \mathrm{eV}$ (red) and $\mathrm{Se} \mathrm{L}_{2,3}$-edges at $1436 \mathrm{eV}$ (green) as well as its composite. (e) XRD pattern of SnSe. (f) Sn 3d and Se 3d regions of the XPS spectrum of SnSe. 
TOP coordinated with Se to yield TOPSe. Without TOP in the precursor solution, SnSe nanostructures could be also obtained, but the decomposition required reaction temperatures above $500{ }^{\circ} \mathrm{C} .{ }^{52}$ Additionally, the aspect ratio of the $\mathrm{SnSe}$ nanostructures produced in the absence of TOP was strongly reduced (Figure S5). When adding small amounts of TOP to the solution, the precursor decomposition temperature could be reduced down to $420{ }^{\circ} \mathrm{C}$ for $\mathrm{V}_{\mathrm{TOP}} / \mathrm{V}_{\mathrm{OAm}}>0.13$, which we associated to the relatively high reactivity of the formed TOPSe. ${ }^{50}$ On the other hand, when replacing OAm by octadecene, SnSe was not formed, probing the important role of $\mathrm{OAm}$ in reducing $\mathrm{SeO}_{2}$ and complexating with $\mathrm{Sn}^{2+}$. Additionally, we tested the influence of the OAm purity on the final product finding no obvious difference in crystal phase and morphology when using two different OAm qualities (80-90\% vs 98\%, Figure S6).
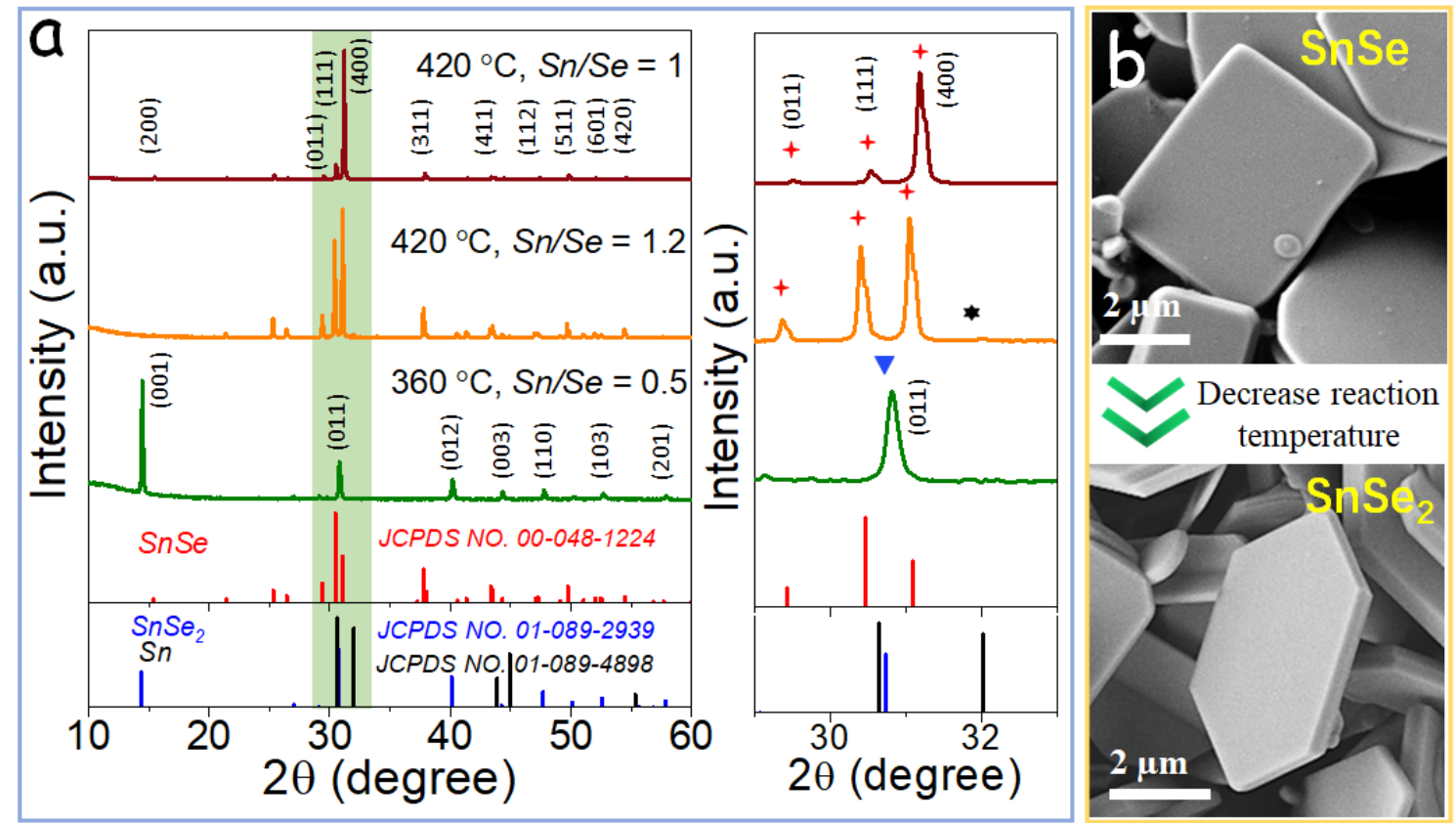

Figure 2. (a) XRD pattern of the powder produced from the decomposition at different temperatures of a precursor containing different ratios of $\mathrm{Sn} / \mathrm{Se}$. A detail of the XRD pattern around $2 \theta=31^{\circ}$ is displayed for clarity. (b) SEM micrographs of SnSe plates with orthorhombic structure and $\mathrm{SnSe}_{2}$ plates with hexagonal phase obtained when decomposing a precursor containing $\mathrm{Sn} / \mathrm{Se}=1$ at $420^{\circ} \mathrm{C}$ and a precursor containing $\mathrm{Sn} / \mathrm{Se}=0.5$ at $360^{\circ} \mathrm{C}$, respectively.

When adding OAc to the precursor ink, a significant change of the geometry of the produced SnSe particles was observed. Figure 3 shows the evolution of the particle shape with the OAc/OAm ratio. We believe the carboxylic group in OAc to have a selective interaction with particular SnSe facets during crystal growth, ${ }^{53,54}$ thus the morphology of SnSe transformed gradually from square shape to dendritic with increasing ratio of OAc/OAm. At 
relatively high $\mathrm{OAc}$ concentrations, $\mathrm{OAc} / \mathrm{OAm}>0.2$, dendritic structures were produced. Through AFM analysis, the thickness of plates and dendritic structures supported on a substrate was measured to be in the range $100-150 \mathrm{~nm}$, which is slightly larger than values obtained from SEM statistics (Figure 3c)

The SnSe powder obtained from the precursor decomposition was thermally annealed at $500{ }^{\circ} \mathrm{C}$ for $60 \mathrm{~min}$ inside a tube furnace with argon flow to remove residual organic ligands. The annealed crystals maintained the original plate geometry and showed no appreciable growth. The annealed powder was subsequently loaded into a graphite die and hot pressed at $500{ }^{\circ} \mathrm{C}$ for 5 minutes under a uniaxial pressure of $80 \mathrm{MPa}$. A cylindrical pellet with a relative density of ca. $95 \%$ was finally obtained (Figure 4a, Table S2). The material cost to produce one cylindrical pellet is estimated in Table S3. 


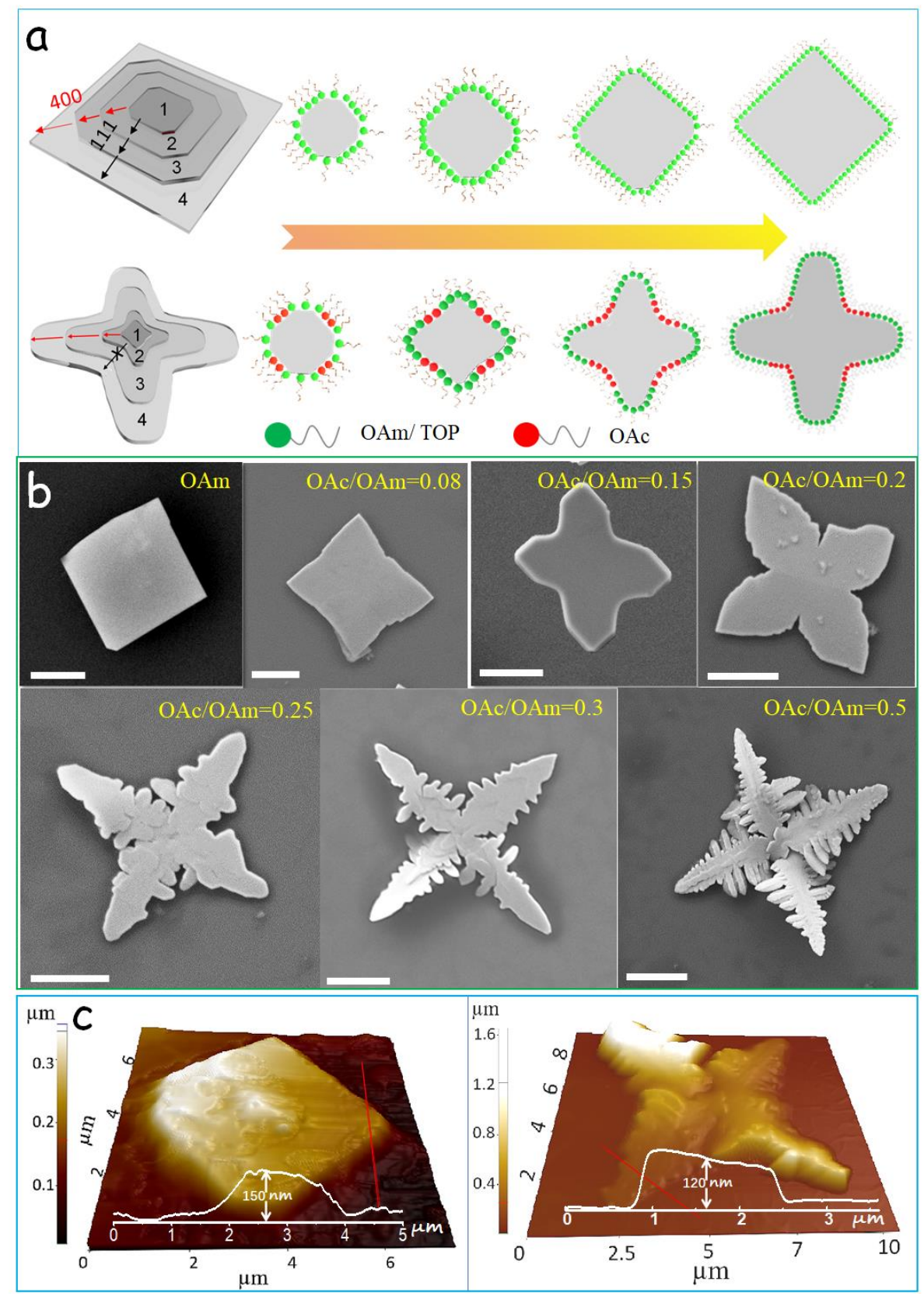

Figure 3. (a) Schematic illustration of the shape evolution for square-like and dendritic crystals. (b) SEM micrographs of the morphology evolution with increasing OAc/OAm ratios. Dendritic nanostructures were obtained when OAc/OAm $>0.2$. Scale bars $=2 \mu \mathrm{m}$. (c) AFM topography images of a square-like and a dendritic nanostructure and height profiles taken from the displayed red line.

The hot pressed material displayed a clear crystallographic texture, with the SnSe [100] crystal direction oriented along the pressure axis, as shown by XRD analysis of the pellet hold with the diffraction plane coincident and normal to the pressure axis (Figure 4b). This crystallographic orientation is consistent with the [100] being the softer crystallographic direction in SnSe. SEM micrographs showed the pellet to have a layered structure, with layers of material assembling normal to the pressure axis, consistent with XRD results (Figure 4c). 
The grain size became slightly larger after the hot-press process as noted by SEM characterization.

To determine the influence of the shape of the SnSe particles on the crystallographic texture of the consolidated material, we hot pressed SnSe particles with $100 \mathrm{~nm}$ average size and $50 \mathrm{~nm}$ thickness produced by a reported method (Figure S9a). ${ }^{31}$ The hot press of these less asymmetric particles in the same conditions as the SnSe plates resulted in polycrystalline materials with no significant texture (Figure S9b,c), proving the influence of the initial particle geometry in the texture of the final polycrystalline material. ${ }^{15,16}$

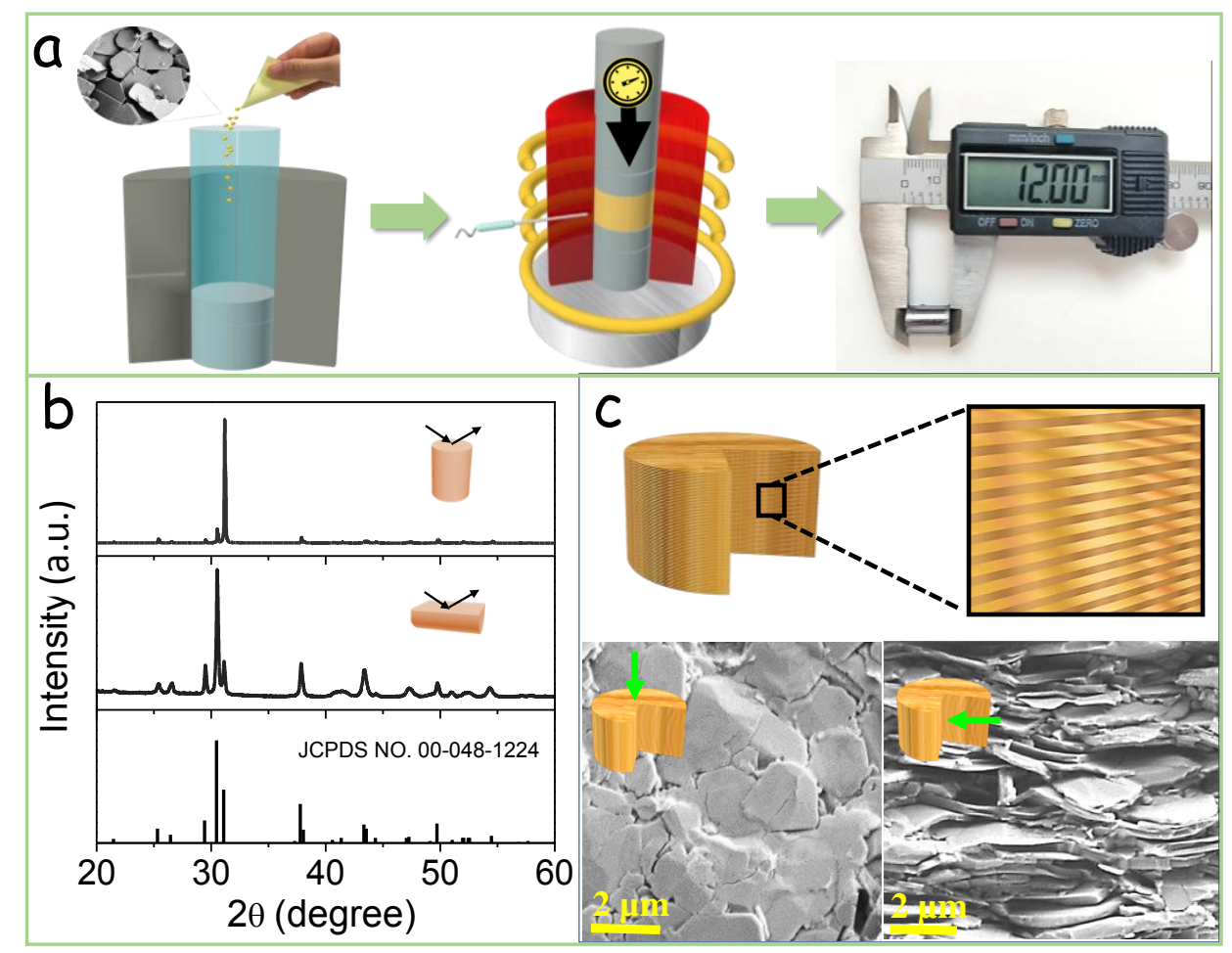

Figure 4. (a) Schematic illustration of the process of consolidation of the SnSe powder into a cylindrical pellet. A SEM micrograph of the annealed SnSe nanopowder and an actual image of consolidated pellet are included in the scheme. (b) XRD pattern of the SnSe pellet laid along the in-plane and cross-plane directions. (c) Representative top view and cross section SEM micrographs of a SnSe pellet.

To demonstrate the potential of the SnSe molecular precursor to produce printed $\mathrm{SnSe}$ layers/patterns, the precursor was printed on a flexible graphite foil using an ink jet nozzle. The graphite foil was then annealed on a hot plate at $420{ }^{\circ} \mathrm{C}$ for $5 \mathrm{~min}$ and additionally hot pressed at $500{ }^{\circ} \mathrm{C}$ for $3 \mathrm{~min}$ under $80 \mathrm{MPa}$ pressure. SEM and XRD analyses of the printed layer demonstrated it to contain SnSe plates oriented parallel to the substrate (Figure S10). 
The TE properties of the polycrystalline SnSe obtained from the precursor decomposition were characterized in two directions (Figure S12): cross plane (//), i.e. parallel to the pressure axis, and in plane $(\perp)$, i.e. normal to pressure axis. Electrical conductivities measured in plane $\left(\sigma_{\perp}\right)$, were higher than those measured cross plane $\left(\sigma_{/ /}\right)$, with $\sigma_{\perp} / \sigma_{/ /}=3.30$. This result is consistent with the much higher charge carrier mobilities in the $b c$ crystal plane of SnSe when compared with its $a$ direction.

Positive Seebeck coefficients were measured in all the temperature range, consistent with the p-type semiconductor character of undoped SnSe. As expected from the minor dependence of this parameter on scattering, similar values of the Seebeck coefficient were measured in both directions, $\mathrm{S}_{\perp} \approx \mathrm{S}_{/ /}$. The slightly higher values systematically obtained cross plane could be ascribed to an energy filtering mechanism at grain boundaries, i.e. a preferential scattering of low energy carriers at the plate interfaces, as previously reported for layered materials. ${ }^{15,16}$

SnSe nanomaterials displayed very low thermal conductivities in both directions, but slightly higher than those obtained from single crystals, most probably due to a certain degree of oxidation. ${ }^{55}$ Significantly lower thermal conductivities were obtained cross plane when compared with in plane, $\kappa_{\perp} / \kappa_{/ /}=1.75$ at $750 \mathrm{~K}$, which was again consistent with the preferential orientation of the plates lying normal to the pressure axis. 

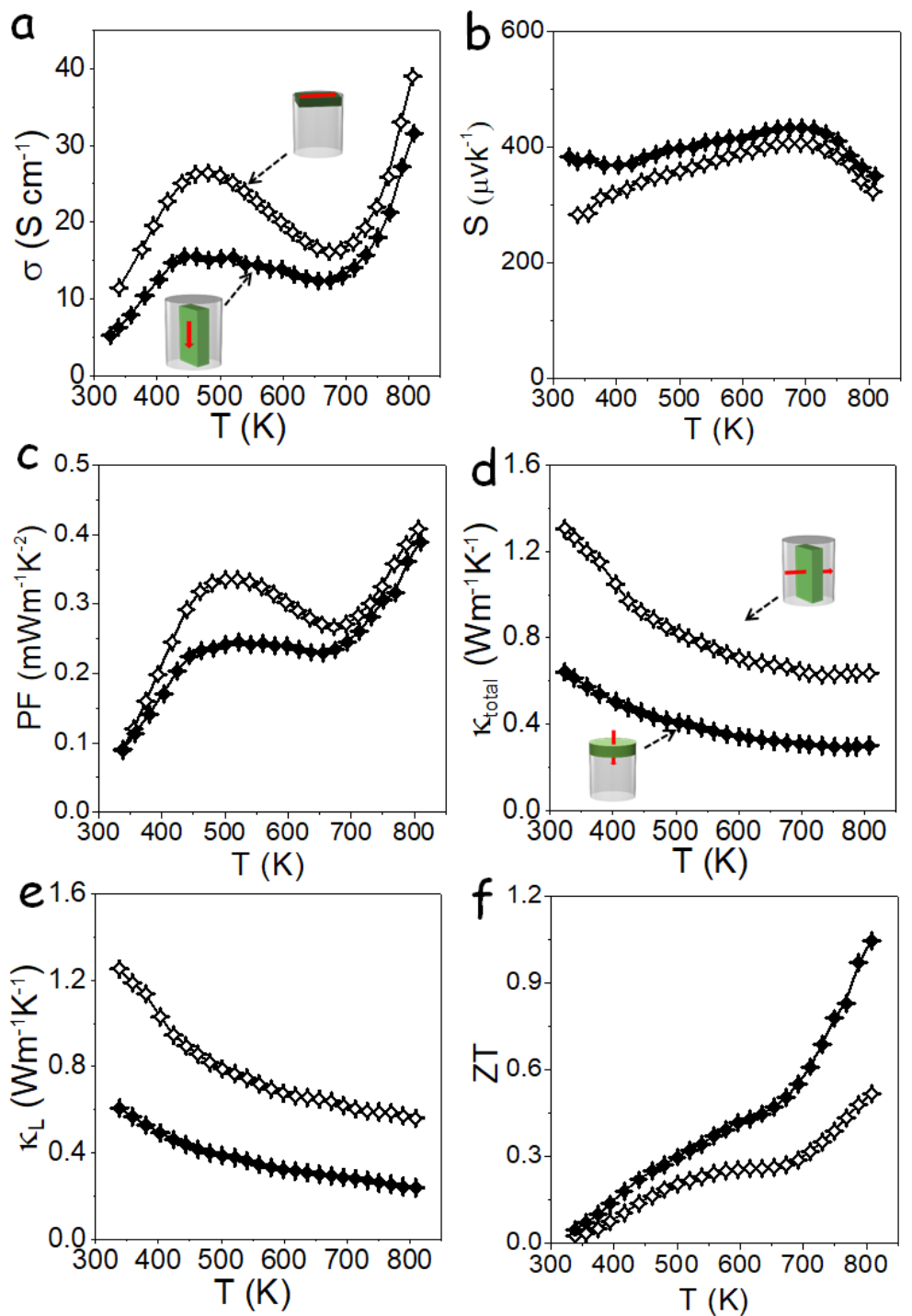

Figure 5. Temperature dependence of (a) electrical conductivity, $\sigma$; (b) Seebeck coefficient, $S$; (c) power factor, $\mathrm{S}^{2} \sigma, \mathrm{PF}$; (d) total thermal conductivity, $\kappa_{\text {total }}$; (e) lattice thermal conductivity, $\kappa_{\mathrm{L}}$; and (f) TE figure of merit, ZT of a $\mathrm{SnSe}_{0.98 \mathrm{Te}} \mathrm{T}_{.02}$ pellet measured in two directions, in-plane ( $\perp$, open symbols) and cross-plane (//, solid symbols).

Overall, the highest $\mathrm{ZT}$ values, up to $\mathrm{ZT}=0.5$ at $770 \mathrm{~K}$, were obtained in plane. This result is consistent with measurements on single crystals and previous reports, ${ }^{13,23,25,56,57}$ but it is unfortunate toward the development of printed SnSe-based modules that target the generation/harvesting of cross plane temperature gradient.

To further promote the TE performance of polycrystalline SnSe layers, small amounts of Te were added to the precursor in replacement of equivalents amounts of Se. The polycrystalline material obtained from the decomposition of the Te-containing precursor, $\mathrm{SnSe}_{1-\mathrm{x}} \mathrm{Te}_{\mathrm{x}}$, was characterized by a slightly reduced band gap (Figure S2), a slightly larger 
charge carrier concentration, $\mathrm{n}_{\mathrm{H}}$, and most important a significantly increased charge carrier mobility, particularly cross plane (Figure S13). Best TE performance was obtained from the material containing a 2 at $\%$ of Te, $\mathrm{SnSe}_{0.98} \mathrm{Te}_{0.2}$ (Figure 5). This material displayed significantly higher electric conductivities than SnSe without a major reduction of the Seebeck coefficient and a minor increase of the total thermal conductivity, associated with the increase of its electronic component. Overall, TE figures of merit up to three-fold larger than those measured for $\mathrm{SnSe}$ were obtained. Most important, the highest TE figures of merit were obtained cross plane, reaching $\mathrm{ZT}=1.05$ at $805 \mathrm{~K}$, which is the highest value reported at this temperature and in this direction for polycrystalline SnSe. This excellent performance was related to the presence of Te facilitating charge transport especially cross plane, which translated into a significant increase of the charge carrier mobility. 


\section{CONCLUSION}

We detailed a fast solution-based approach to produce crystallographic textured SnSe bulk nanomaterials and printed layers. The molecular precursor solution was prepared directly by dissolving ionic Sn and Se species in a thiol-free and nontoxic solvent. Pure phase SnSe powder was obtained by decomposing the molecular precursor at $420{ }^{\circ} \mathrm{C}$. The composition, crystal phase and shape of the produced SnSe nanostructures could be tuned by modifying the nominal elemental ratio, the decomposition temperature and the use of additional surfactants. Crystallographic textured SnSe bulk nanomaterials and layers were obtained after hot-pressing the precursor decomposition products. These materials showed moderate TE performances cross plane which was unfortunate for the design a TE devices that generate/harvest temperature gradients in the direction normal to the substrate. The SnSe TE performance was significantly improved, up to a threefold, by introducing small amounts of Te during precursor formulation. The presence of this small amount of Te resulted in an important increase of electrical conductivity cross plane associated to an increase of charge carrier concentration and especially mobility with a minor variation of the Seebeck coefficient. The presence of 2 at $\%$ of Te in $\mathrm{SnSe}_{0.98} \mathrm{Te}_{0.02}$ finally translated into record cross plane $\mathrm{ZT}$ values at $800 \mathrm{~K}$ for polycrystalline $\mathrm{SnSe}$.

\section{EXPERIMENTAL SECTION}

Chemicals: Tin chloride $\left(\mathrm{SnCl}_{2}, 98 \%\right)$ and selenium dioxide $\left(\mathrm{SeO}_{2}, 99.8 \%\right)$ were purchased from Acros Organics. Tri-n-octylphosphine (TOP, $\mathrm{C}_{24} \mathrm{H}_{51} \mathrm{P}, 97 \%$ ), oleic acid $\left(\mathrm{OAc}, \mathrm{C}_{18} \mathrm{H}_{34} \mathrm{O}_{2}\right.$, $90 \%)$ and oleylamine (OAm, $\left.\mathrm{C}_{18} \mathrm{H}_{37} \mathrm{~N}, \geq 98 \%\right)$ were purchased from Sigma-Aldrich. Sodium tellurite $\left(\mathrm{Na}_{2} \mathrm{TeO}_{3}, 99.5 \%\right)$ was purchased from Fisher. Analytical grade chloroform and ethanol were ordered from various sources. All chemicals were used as received unless specifically noted.

SnSe precursor: Within an argon-filled glove box, $5.76 \mathrm{~g} \mathrm{SnCl}_{2}(30 \mathrm{mmol})$ and $3.33 \mathrm{~g} \mathrm{\textrm {SeO } _ { 2 }}$ (30 mmol) were weighted and placed inside a glass bottle. Then $150 \mathrm{~mL}$ OAm and $20 \mathrm{ml}$ TOP were added inside the bottle. The mixture was subsequently sonicated for $20 \mathrm{~min}$ until precursors dissolved completely. Small amounts of OAc were included within the precursor to produce dentritic SnSe nanostructures. 
SnSe 1-x $_{\text {Tex }}$ precursor: To produce $\mathrm{SnSe}_{1-\mathrm{x}} \mathrm{Te}_{\mathrm{x}}(\mathrm{x}=0.01,0.02,0.03)$ precursor precursors, 5.76 $\mathrm{g} \mathrm{SnCl}_{2}(30 \mathrm{mmol}), 3.33 \mathrm{~g} \mathrm{SeO} 2(30 \mathrm{mmol})$ and $6.65 \mathrm{x} \mathrm{g} \mathrm{Na}_{2} \mathrm{TeO}_{3}$ were weighted and placed in a glass bottle, where $150 \mathrm{~mL}$ OAm and $20 \mathrm{ml}$ TOP was subsequently added. The mixture was sonicated for 20 min until all precursors dissolved completely.

SnSe precursor decomposition: The SnSe precursor was continuously injected into a preheated glass flask $\left(420^{\circ} \mathrm{C}\right)$, where it immediately decomposed. The flask was naturally cooled down to room temperature (RT) and the crystalline product was collected by dispersion in chloroform and precipitation with ethanol. Dispersion/precipitation steps were repeated three times using chloroform and ethanol aided by centrifugation at $7200 \mathrm{rpm}$ for $5 \mathrm{~min}$. The produced SnSe material was kept inside glovebox for further use.

Bulk material consolidation: SnSe particles were thermally annealed at $500{ }^{\circ} \mathrm{C}$ (heating rate $=10{ }^{\circ} \mathrm{C} / \mathrm{min}$ ) for $60 \mathrm{~min}$ inside a tube furnace with argon flow to remove organic residues. After cooling to RT, the annealed material was grounded in a mortar and subsequently loaded into a graphite die. The die was then transferred to a custom-made hot press system inside the glove box, where it was heated to $500{ }^{\circ} \mathrm{C}$ by using an induction coil. The die was held at this temperature for $5 \mathrm{~min}$ under $80 \mathrm{MPa}$ pressure. Afterward, the pressure was released and the die was naturally cooled down to RT. The obtained cylindrical pellets ( $\varnothing 8 \mathrm{~mm} \times 12 \mathrm{~mm}$ ) were cut in rectangular bars in two directions: along the pressure axis (cross-plane direction) and normal to this axis (in-plane direction). The consolidated pellets were subsequently kept inside glovebox. Before performing measurement, the pellets were polished slightly to remove a possible surface oxide layer.

Printed layers: The SnSe molecular precursor was printed on a graphite foil using an ink jet nozzle. The graphite foil was then annealed on a hot plate at $420{ }^{\circ} \mathrm{C}$ for $5 \mathrm{~min}$ within an argonfilled glove box. Afterward, the foil was hot pressed at $500{ }^{\circ} \mathrm{C}$ for $3 \mathrm{~min}$ under $80 \mathrm{MPa}$ pressure.

Structural and chemical characterization: The particle size and morphology were characterized by transmission electron microscopy (TEM, ZEISS LIBRA 120), working at 120 $\mathrm{kV}$ and field-emission scanning electron microscopy (SEM, Zeiss Auriga) operating at $5.0 \mathrm{kV}$. High resolution TEM (HRTEM) images and scanning TEM (STEM) studies were conducted on a FEI Tecnai F20 field emission gun microscope operated at $200 \mathrm{kV}$ with a point-to-point resolution of $0.19 \mathrm{~nm}$, which was equipped with high angle annular dark field (HAADF) and Gatan Quantum electron energy loss spectroscopy (EELS) detectors. Elemental analysis was performed using an Oxford energy dispersive X-ray spectrometer (EDX) combined with the 
Zeiss Auriga SEM working at $20.0 \mathrm{kV}$. X-ray diffraction analyses (XRD, 20: $5^{\circ}-80^{\circ}$, scanning rate was set at $5 \% \mathrm{~min}$ ) were carried out on a Bruker AXS D8 Advance X-ray diffractometer with Ni-filtered $\mathrm{Cu}-\mathrm{K} \alpha$ radiation $(\lambda=1.5406 \AA)$, operating at $40 \mathrm{~mA}$ and $40 \mathrm{kV}$. X-ray photoelectron spectroscopy (XPS) was performed on a SPECS system equipped with a Phoibos 150 MCD-9 detector, working at $150 \mathrm{~W}$ with an Al anode XR50 source. Fourier transform infrared spectroscopy (FTIR, Alpha Bruker) was carried out with a platinum attenuated total reflectance single reflection module. The surface topology was measured by atomic force microscopy (AFM, XE 100 Park System Corp.) Scans were conducted in non-contact mode with a silicon Tap300Al-G cantilever (Budget Sensors, spring constant of $\sim 40 \mathrm{~N} / \mathrm{m}$ and resonant frequency of $\sim 300 \mathrm{kHz}$ ). AFM images were analysed in the XEI software (Park System Corp). Ultraviolet-Visible Spectrophotometry (UV-Vis) Optical absorption spectra were recorded on a LAMBDA $950 \mathrm{UV}-\mathrm{Vis}$ spectrophotometer from PerkinElmer.

Thermoelectric characterization: Seebeck coefficients were measured with a static DC method. Electric resistivity data was obtained using a standard four probe method. Electric resistivity and Seebeck coefficients were measured simultaneously in a LSR-3 LINSEIS system in the temperature range from 323 to $803 \mathrm{~K}$ under a helium atmosphere. Bearing in mind the measurement precision and system accuracy, an error of ca. 5\% in the measurement of Seebeck coefficient and electrical conductivity was estimated. A Xenon Flash Apparatus was used to measure the thermal diffusivities (D) of all samples with accuracies better than $6 \%$. Thermal conductivities were then calculated by the relation $\kappa=C_{p} D \rho$, where $C_{p}$ is the heat capacity that was estimated from the Dulong-Petit law, D is the measured thermal diffusivity and $\rho$ is the mass density that was measured using the Archimedes' method. Hall charge carrier concentrations $\left(\mathrm{n}_{\mathrm{H}}\right)$ and mobilities $\left(\mu_{\mathrm{H}}\right)$ at room temperature $(300 \mathrm{~K})$ were measured with a on Van der Pauw and Hall Bar measurements (ezHEMS 1000, NanoMagnetics) using a magnetic field of $1 \mathrm{~T}$. Values provided correspond to the average of 10 consecutive measurements, from which an error of ca. $10 \%$ was estimated.

\section{ASSOCIATED CONTENT}

Supporting Information. Supporting information contains precursor formation mechanism, UV-vis spectra, SEM-EDX, electrical transport properties, stability and reproducibility test.

\section{AUTHOR INFORMATION}




\section{Corresponding Authors}

* A. Cabot: acabot@irec.cat

* M. Ibañez: mibanez@ist.ac.at

\section{ORCID}

Yu Zhang: 0000-0002-0332-0013

Yu Liu: 0000-0001-7313-6740

Jordi Arbiol: 0000-0002-0695-1726

Doris Cadavid: 0000-0002-1376-6078

Maria Ibáñez: 0000-0001-5013-2843

Andreu Cabot: 0000-0002-7533-3251

\section{AUTHOR CONTRIBUTIONS}

\# Y. Zhang and Y. Liu contributed equally to this work. The manuscript was written through contributions of all authors. All authors have given approval to the final version of the manuscript.

\section{NOTES}

The authors declare no competing financial interest.

\section{ACKNOWLEDGMENTS}

MI acknowledges financial support from IST Austria. YL acknowledges funding from the European Union's Horizon 2020 research and innovation programme under the Marie Sklodowska-Curie grant agreement No. 754411. JL is a Serra Húnter Fellow and is grateful to ICREA Academia program and projects MICINN/FEDER RTI2018-093996-B-C31 and GC 2017 SGR 128. This work was supported by the European Regional Devel-opment Funds and by the Spanish Ministerio de Economía y Com-petitividad through the project SEHTOP (ENE2016-77798-C4-3-R). Y.Z., C.X., M.L., X.Y., and T.Z. thank the China Scholarship Council for the scholarship support. ICN2 acknowledges funding from Generalitat de Catalunya 2017 SGR 327 and the Spanish MINECO project ENE2017-85087-C3. ICN2 is supported by the Severo Ochoa program from the Spanish MINECO (grant no. SEV-20170706) and is funded by the CERCA Pro-gramme/Generalitat de Catalunya. Part of the present 
work has been performed in the framework of Universitat Autònoma de Barcelona Materials Science PhD program.

\section{REFERENCE}

1. Bell, L. E., Cooling, Heating, Generating Power, and Recovering Waste Heat with Thermoelectric Systems. Science 2008, 321(5895), 1457-1461.

2. Sootsman, J. R.; Chung, D. Y.; Kanatzidis, M. G., New and Old Concepts in Thermoelectric Materials. Angew. Chem. Int. Ed. 2009, 48(46), 8616-8639.

3. Snyder, G.; Toberer, E., Complex Thermoelectric Materials. Nat. Mater. 2008, 7, 105-114.

4. Minnich, A.; Dresselhaus, M.; Ren, Z.; Chen, G., Bulk Nanostructured Thermoelectric Materials: Current Research and Future Prospects. Energy Environ. Sci. 2009, 2(5), 466479.

5. Leonov, V.; Torfs, T.; Fiorini, P.; Van Hoof, C., Thermoelectric Converters of Human Warmth for Self-powered Wireless Sensor Nodes. IEEE Sens. J. 2007, 7(5), 650-657.

6. Kraemer, D.; Poudel, B.; Feng, H.-P.; Caylor, J. C.; Yu, B.; Yan, X.; Ma, Y.; Wang, X.; Wang, D.; Muto, A.; Kenneth, M,; Matteo, C,; Chen, G,; Ren, Z., High-performance FlatPanel Solar Thermoelectric Generators with High Thermal Concentration. Nat. Mater. 2011, 10(7), 532-538.

7. Ortega, S.; Ibáñez, M.; Liu, Y.; Zhang, Y.; Kovalenko, M. V.; Cadavid, D.; Cabot, A., Bottom-Up Engineering of Thermoelectric Nanomaterials and Devices From Solutionprocessed Nanoparticle Building Blocks. Chem. Soc. Rev. 2017, 46(12), 3510-3528.

8. Ibáñez, M.; Luo, Z.; Genc, A.; Piveteau, L.; Ortega, S.; Cadavid, D.; Dobrozhan, O.; Liu, Y.; Nachtegaal, M.; Zebarjadi, M.; Arbiol, J.; Kovalenko, M. V.; Cabot, A., Highperformance Thermoelectric Nanocomposites from Nanocrystal Building Blocks. Nat. Commun. 2016, 7, 10766.

9. Snyder, G. J.; Ursell, T. S., Thermoelectric Efficiency and Compatibility. Phys. Rev. Lett. 2003, 91 (14), 148301.

10. Ibáñez, M.; Hasler, R.; Genç, A.; Liu, Y.; Kuster, B.; Schuster, M.; Dobrozhan, O.; Cadavid, D.; Arbiol, J.; Cabot, A.; M, Kovalenko., Ligand-Mediated Band Engineering in Bottom-Up Assembled SnTe Nanocomposites for Thermoelectric Energy Conversion. $J$. Am. Chem. Soc. 2019. 141(20), 8025-8029.

11. Zhou, Y.; Zhao, L. D., Promising Thermoelectric Bulk Materials with 2D Structures. Adv. Mater. 2017, 29(45), 1702676.

12. Dresselhaus, M.; Dresselhaus, G.; Sun, X.; Zhang, Z.; Cronin, S.; Koga, T., Lowdimensional Thermoelectric Materials. Phys. Solid State 1999, 41(5), 679-682.

13. Zhao, L.-D.; Lo, S.-H.; Zhang, Y.; Sun, H.; Tan, G.; Uher, C.; Wolverton, C.; Dravid, V. P.; Kanatzidis, M. G., Ultralow Thermal Conductivity and High Thermoelectric Figure of Merit in SnSe Crystals. Nature 2014, 508 (7496), 373-377.

14. Rhyee, J. S.; Ahn, K.; Lee, K. H.; Ji, H. S.; Shim, J. H., Enhancement of the Thermoelectric Figure $\square$ of $\square$ Merit in a Wide Temperature Range in $\operatorname{In}_{4} \mathrm{Se}_{3-\mathrm{x}} \mathrm{Cl}_{0.03}$ Bulk Crystals. Adv. Mater. 2011, 23(19), 2191-2194.

15. Liu, Y.; Zhang, Y.; Lim, K. H.; Ibáñez, M.; Ortega, S.; Li, M.; David, J.; Martí-Sánchez, S.; Ng, K. M.; Arbiol, J.; Kovalenko, M. V.; Cadavid, D.; Cabot, High Thermoelectric 
Performance in Crystallographically Textured n-Type $\mathrm{Bi}_{2} \mathrm{Te}_{3-\mathrm{x}} \mathrm{Se}_{\mathrm{x}}$ Produced from Asymmetric Colloidal Nanocrystals. ACS nano 2018, 12 (7), 7174-7184.

16. Liu, Y.; Zhang, Y.; Ortega, S.; Ibáñez, M.; Lim, K. H.; Grau-Carbonell, A.; Martí-Sánchez, S.; Ng, K. M.; Arbiol, J.; Kovalenko, M. V.; Cadavid, D.; Cabot, A.Crystallographically Textured Nanomaterials Produced from the Liquid Phase Sintering of $\mathrm{Bi}_{\mathrm{x}} \mathrm{Sb}_{2-\mathrm{x}} \mathrm{Te}_{3}$ Nanocrystal Building Blocks. Nano Lett. 2018, 18(4), 2557-2563.

17. Dun, C.; Liu, Y.; Al-Qawasmeh, A.; Hewitt, C. A.; Guo, Y.; Xu, J.; Jiang, Q.; Wang, J.; Marcus, G.; Cadavid, D.; Montgomery, D.; Wang, H.; Kovnir, K.; Cabot, A., Carroll, D. L., Topological Doping Effects in 2D Chalcogenide Thermoelectrics. 2D Materials 2018, 5 (4), 045008.

18. Poudel, B.; Hao, Q.; Ma, Y.; Lan, Y.; Minnich, A.; Yu, B.; Yan, X.; Wang, D.; Muto, A.; Vashaee, D., Xiaoyuan, C; Liu, J,; Mildred, D,; Chen, G,; Ren, Z., High-Thermoelectric Performance of Nanostructured Bismuth Antimony Telluride Bulk Aloys. Science 2008, 320 (5876), 634-638.

19. Zhao, X. B.; Ji, X. H.; Zhang, Y. H.; Zhu, T. J.; Tu, J. P.; Zhang, X. B., Bismuth Telluride Nanotubes and the Effects on the Thermoelectric Properties of Nanotube-Containing Nanocomposites. Appl. Phys. Lett. 2005, 86(6), 062111.

20. Yamashita, O.; Tomiyoshi, S.; Makita, K., Bismuth Telluride Compounds with High Thermoelectric Figures of Merit. J. Appl. Phys. 2003, 93(1), 368-374.

21. Goldsmid, H. J., Bismuth Telluride and Its Alloys as Materials for Thermoelectric Generation. Materials 2014, 7(4), 2577-2592.

22. Chang, C.; Wu, M.; He, D.; Pei, Y.; Wu, C.-F.; Wu, X.; Yu, H.; Zhu, F.; Wang, K.; Chen, Y.; Huang, L.; Li, J.-F.; He, J.; Zhao, L.-D., 3D Charge and 2D Phonon Transports Leading to High Out-of-plane ZT in n-type SnSe Crystals. Science 2018, 360(6390), 778-783.

23. Chen, C.-L.; Wang, H.; Chen, Y.-Y.; Day, T.; Snyder, G. J., Thermoelectric Properties of P-type Polycrystalline SnSe Doped with Ag. J. Mater. Chem. A 2014, 2(29), 11171-11176.

24. Sassi, S.; Candolfi, C.; Vaney, J.-B.; Ohorodniichuk, V.; Masschelein, P.; Dauscher, A.; Lenoir, B., Assessment of the Thermoelectric Performance of Polycrystalline p-type SnSe. Appl. Phys. Lett. 2014, 104(21), 212105.

25. Wei, T.-R.; Tan, G.; Zhang, X.; Wu, C.-F.; Li, J.-F.; Dravid, V. P.; Snyder, G. J.; Kanatzidis, M. G., Distinct Impact of Alkali-ion Doping on Electrical Transport Properties of Thermoelectric p-type Polycrystalline SnSe. J. Am. Chem. Soc. 2016, 138(28), 8875-8882.

26. Chere, E. K.; Zhang, Q.; Dahal, K.; Cao, F.; Mao, J.; Ren, Z., Studies on Thermoelectric Figure of Merit of Na-doped p-type Polycrystalline SnSe. J. Mater. Chem. A 2016, 4(5), 1848-1854.

27. Shi, X.; Wu, A.; Liu, W.; Moshwan, R.; Wang, Y.; Chen, Z.-G.; Zou, J., Polycrystalline SnSe with Extraordinary Thermoelectric Property via Nanoporous Design. ACS nano 2018, 12 (11), 11417-11425.

28. Chandra, S.; Banik, A.; Biswas, K., N-Type Ultrathin Few-Layer Nanosheets of Bi-doped SnSe: Synthesis and Thermoelectric Properties. ACS Energy Lett. 2018, 3(5), 1153-1158.

29. Ananya, B.; Biswas, K., A game-changing strategy in SnSe thermoelectrics. Joule. 2019, 3(3), 636-638.

30. Chandra, S.; Biswas, K., Realization of high thermoelectric figure of merit in solution synthesized 2D SnSe nanoplates via Ge alloying. J. Am. Chem. Soc. 2019, 141 (15), 61416145. 
31. Han, G.; Popuri, S. R.; Greer, H. F.; Bos, J. W. G.; Zhou, W.; Knox, A. R.; Montecucco, A.; Siviter, J.; Man, E. A.; Macauley, M.; Paul, D. J.; Li, W. -G.; Paul, M. C.; Gao, M.; Sweet, T.; Freer, R.; Azough, F.; Baig, H.; Sellami, N.; Mallick, T.; K.; Gregory, D. H., Facile Surfactant $\square$ Free Synthesis of $\mathrm{p} \square$ Type SnSe Nanoplates with Exceptional Thermoelectric Power Factors. Angew. Chem. Int. Ed. 2016, 55(22), 6433-6437.

32. Ge, Z. H.; Qiu, Y.; Chen, Y. X.; Chong, X.; Feng, J.; Liu, Z. K.; He, J., Multipoint Defect Synergy Realizing the Excellent Thermoelectric Performance of $\mathrm{n} \square$ Type Polycrystalline SnSe via Re Doping. Adv. Funct. Mater. 2019, 1902893.

33. Luo, Y.; Cai, S.; Hua, X.; Chen, H.; Liang, Q.; Du, C.; Zheng, Y.; Shen, J.; Xu, J.; Wolverton, C., Dravid, V.; Yan, Q.; Kanatzidis, M. G., High Thermoelectric Performance in Polycrystalline SnSe Via Dual $\square$ Doping with $\mathrm{Ag} / \mathrm{Na}$ and Nanostructuring with $\mathrm{Ag}_{8} \mathrm{SnSe}_{6}$. Adv. Energy Mater. 2019, 9(2), 1803072.

34. Shi, X.; Wu, A.; Feng, T.; Zheng, K.; Liu, W.; Sun, Q.; Hong, M.; Pantelides, S. T.; Chen, Z. G.; Zou, J., High Thermoelectric Performance in $\mathrm{p} \square$ type Polycrystalline Cd $\square$ doped SnSe Achieved by a Combination of Cation Vacancies and Localized Lattice Engineering. Adv. Energy Mater. 2019, 9(11), 1803242.

35. Heo, S.; H., Jo, S.; Kim, H. S.; Choi, G.; Song, J. Y.; Kang, J. Y.; Park, N.; Ban, H.W.; Kim, F.; Jeong, H.; Jung, J.; Jang, J.; Lee, W.; Shin, H.; Son, J. S., Composition changedriven texturing and doping in solution-processed SnSe thermoelectric thin films. Nat. Commun. 2019, 10 (1), 1-10.

36. Ashida, M.; Hamachiyo, T.; Hasezaki, K.; Matsunoshita, H.; Kai, M.; Horita, Z., Texture of Bismuth Telluride-based Thermoelectric Semiconductors Processed by High-pressure Torsion. J. Phys. Chem. Solids 2009, 70(7), 1089-1092.

37. Rogl, G.; Setman, D.; Schafler, E.; Horky, J.; Kerber, M.; Zehetbauer, M.; Falmbigl, M.; Rogl, P.; Royanian, E.; Bauer, E., High-pressure Torsion, A New Processing Route for Thermoelectrics of High ZTs by Means of Severe Plastic Deformation. Acta Mater. 2012, 60(5), 2146-2157.

38. Angerer, P.; Neubauer, E.; Yu, L.; Khor, K. A., Texture and Structure Evolution of Tantalum Powder Samples During Spark-Plasma-Sintering (SPS) and Conventional Hot-pressing. Int. J. Refract. Met. H. 2007, 25(4), 280-285.

39. Jiang, J.; Chen, L.; Bai, S.; Yao, Q.; Wang, Q., Fabrication and Thermoelectric Performance of Textured n-type $\mathrm{Bi}_{2}(\mathrm{Te}, \mathrm{Se})_{3}$ by Spark Plasma Sintering. Mater. Sci. Eng. B 2005, 117(3), 334-338.

40. Jiang, J.; Chen, L.; Bai, S.; Yao, Q.; Wang, Q., Thermoelectric Properties of Textured Ptype (Bi, Sb) ${ }_{2} \mathrm{Te}_{3}$ Fabricated by Spark Plasma Sintering. Scr. Mater. 2005, 52(5), 347-351.

41. Li, M.; Liu, Y.; Zhang, Y.; Zuo, Y.; Li, J.; Lim, K. H.; Cadavid, D.; Ng, K. M.; Cabot, A., Crystallographically Textured SnSe Nanomaterials Produced from the Liquid Phase Sintering of Nanocrystals. Dalton Trans. 2019, 48(11), 3641-3647.

42. Zhang, J.; Xu, J.; Tan, X.; Wang, H.; Liu, G.-Q.; Shao, H.; Yu, B.; Yue, S.; Jiang, J., Optimized Orientation and Enhanced Thermoelectric Performance in $\mathrm{Sn}_{0.97} \mathrm{Na}_{0.03} \mathrm{Se}$ with Te Addition. J. Mater. Chem. C 2019, 7(9), 2653-2658.

43. Liu, X.; Li, Y.; Zhou, B.; Wang, X.; Cartwright, A. N.; Swihart, M. T. Shape-controlled synthesis of $\mathrm{SnE}(\mathrm{E}=\mathrm{S}, \mathrm{Se})$ semiconductor nanocrystals for optoelectronics. Chemistry of Materials 2014, 26 (11), 3515-3521. 
44. Li, L.; Chen, Z.; Hu, Y.; Wang, X.; Zhang, T.; Chen, W.; Wang, Q. Single-layer singlecrystalline SnSe nanosheets. Journal of the American Chemical Society 2013, 135 (4), 1213-1216.

45. Liu, S.; Guo, X.; Li, M.; Zhang, W. H.; Liu, X.; Li, C. Solution- phase synthesis and characterization of single- crystalline SnSe nanowires. Angewandte Chemie International Edition 2011, 50 (50), 12050-12053.

46. Baumgardner, W. J.; Choi, J. J.; Lim, Y.-F.; Hanrath, T. SnSe nanocrystals: synthesis, structure, optical properties, and surface chemistry. Journal of the American Chemical Society 2010, 132 (28), 9519-9521.

47. Ning, J.; Xiao, G.; Jiang, T.; Wang, L.; Dai, Q.; Zou, B.; Liu, B.; Wei, Y.; Chen, G.; Zou, G. Shape and size controlled synthesis and properties of colloidal IV-VI SnSe nanocrystals. CrystEngComm 2011, 13 (12), 4161-4166.

48. Yarema, M.; Caputo, R.; Kovalenko, M. V., Precision Synthesis of Colloidal Inorganic Nanocrystals Using Metal and Metalloid Amides. Nanoscale 2013, 5 (18), 8398-8410.

49. Kravchyk, K.; Protesescu, L.; Bodnarchuk, M. I.; Krumeich, F.; Yarema, M.; Walter, M.; Guntlin, C.; Kovalenko, M. V., Monodisperse and Inorganically Capped Sn and $\mathrm{Sn} / \mathrm{SnO} 2$ Nanocrystals for High-performance Li-ion Battery Anodes. J. Am. Chem. Soc. 2013, 135(11), 4199-4202.

50. Mourdikoudis, S.; Liz-Marzan, L. M., Oleylamine in Nanoparticle Synthesis. Chem. Mater. 2013, 25 (9), 1465-1476.

51. Evans, C. M.; Evans, M. E.; Krauss, T. D., Mysteries of TOPSe Revealed: Insights into Quantum Dot Nucleation. J. Am. Chem. Soc. 2010, 132(32), 10973-10975.

52. Zhang, Y.; Liu, Y.; Lim, K. H.; Xing, C.; Li, M.; Zhang, T.; Tang, P.; Arbiol, J.; Llorca, J.; Ng, K. M.; Ibáñez, M.; Guardia, P.; Prato, M.; Cadavid, D.; Cabot, A.,Tin Diselenide Molecular Precursor for Solution $\square$ Processable Thermoelectric Materials. Angew. Chem. Int. Ed. 2018, 130(52), 17309-17314.

53. Berestok, T.; Guardia, P.; Blanco, J.; Nafria, R.; Torruella, P.; Lopez-Conesa, L.; Estrade, S.; Ibáñez, M.; De Roo, J.; Luo, Z.; Cadavid, D.; Martins, J. C.; Kovalenko, M. V.; Peiró, F.; Cabot, A., Tuning Branching in Ceria Nanocrystals. Chem. Mater. 2017, 29(10), 44184424.

54. Watt, J.; Cheong, S.; Toney, M. F.; Ingham, B.; Cookson, J.; Bishop, P. T.; Tilley, R. D., Ultrafast Growth of Highly Branched Palladium Nanostructures for Catalysis. ACS nano 2010, 4 (1), 396-402.

55. Lee, Y. K.; Luo, Z.; Cho, S. P.; Kanatzidis, M. G.; Chung, I., Surface Oxide Removal for Polycrystalline SnSe Reveals Near-Single-Crystal Thermoelectric Performance. Joule 2019, 3(3), 719-731.

56. Shi, X.; Zheng, K.; Hong, M.; Liu, W.; Moshwan, R.; Wang, Y.; Qu, X.; Chen, Z.-G.; Zou, J., Boosting the Thermoelectric Performance of P-type heavily Cu-doped Polycrystalline SnSe via Inducing Intensive Crystal Imperfections and Defect Phonon Scattering. Chem. Sci. 2018, 9(37), 7376-7389.

57. Ge, Z.-H.; Song, D.; Chong, X.; Zheng, F.; Jin, L.; Qian, X.; Zheng, L.; Dunin-Borkowski, R. E.; Qin, P.; Feng, J.; Zhao, L.-D., Boosting the Thermoelectric Performance of (Na, K)Codoped Polycrystalline SnSe by Synergistic Tailoring of the Band Structure and AtomicScale Defect Phonon Scattering. J. Am. Chem. Soc. 2017, 139(28), 9714-9720. 
Table of content

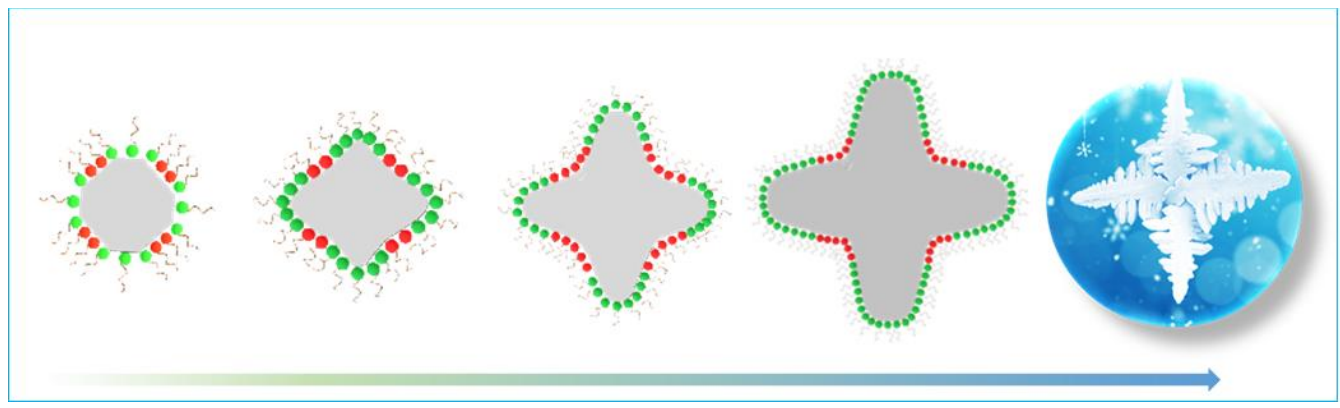

